University of Nebraska - Lincoln

DigitalCommons@University of Nebraska - Lincoln

6-1-1998

\title{
Finite size scaling in the thin film limit
}

\author{
C. Waldfield \\ University of Nebraska-Lincoln \\ D. Welipitiya \\ University of Nebraska-Lincoln \\ T. McAvoy \\ University of Nebraska-Lincoln \\ Peter A. Dowben \\ University of Nebraska-Lincoln, pdowben@unl.edu \\ Elio Vescovo \\ Brookhaven National Laboratory, vescovo@bnl.gov
}

Follow this and additional works at: https://digitalcommons.unl.edu/physicsdowben

Part of the Physics Commons

Waldfield, C.; Welipitiya, D.; McAvoy, T.; Dowben, Peter A.; and Vescovo, Elio, "Finite size scaling in the thin film limit" (1998). Peter Dowben Publications. 38.

https://digitalcommons.unl.edu/physicsdowben/38

This Article is brought to you for free and open access by the Research Papers in Physics and Astronomy at DigitalCommons@University of Nebraska - Lincoln. It has been accepted for inclusion in Peter Dowben Publications by an authorized administrator of DigitalCommons@University of Nebraska - Lincoln. 


\title{
Finite size scaling in the thin film limit
}

\author{
C. Waldfried, D. Welipitiya, T. McAvoy, and P. A. Dowben \\ Department of Physics \& Astronomy and the Center for Materials Research and Analysis, \\ University of Nebraska-Lincoln, Lincoln, Nebraska 68588-0111
}

\section{E. Vescovo}

National Synchrotron Light Source, Brookhaven National Laboratory, Upton, New York 11973

The thickness dependent spin-polarized electronic structure of strained thin and ultrathin films of Gd is characterized by a distinct change in the critical exponent $\lambda$ in the formalism of finite size scaling. The reduced critical exponent in the ultrathin films can be correlated to the increased dominance of the surface magnetic structure and the increasing paramagnetic-like behavior of the bulk. (C) 1998 American Institute of Physics. [S0021-8979(98)34111-0]

With decreasing film thickness the surface may become increasingly more important for the magnetic ordering of ultrathin films. The surface and bulk electronic structure may adopt the role of "actor" or "spectator" in a ferromagnetic thin film. ${ }^{1}$ Electronic structure and its influence will depend in any case, upon electron wave vector ${ }^{2}$ and the film thickness. $^{3}$

The distinct magnetic properties of the surface as well as the bulk will have significant effects on the finite size scaling behavior of thin films and deviations from the expected finite size scaling behavior ${ }^{4}$

$$
\frac{T_{C}(\infty)-T_{C}(d)}{T_{C}(\infty)}=C \cdot d^{-\lambda}
$$

can be expected. ${ }^{3}$ If surface magnetism has a prominent "active" role in the magnetic ordering of ultrathin films it will influence the critical exponent $\lambda$. There have been a few examples of deviations in the critical exponent $\lambda$ in the thin film limit to date. ${ }^{5}$

We studied the finite size scaling behavior for increasingly thinner films of strained $\mathrm{Gd}^{6,7}$ with spin-polarized photoemission as described elsewhere. ${ }^{2,3}$ The spin-polarized valence-band photoemission spectra of the strained Gd films have distinct features attributable to the surface and the bulk. ${ }^{2,3,8}$ This permits one to characterize the magnetic behavior of the surface and the bulk separately, ${ }^{2,3}$ as has been done for "unstrained" $\mathrm{Gd}(0001) .{ }^{9}$ Strained films of gadolinium with an increased lattice constant of approximately $4 \%$ have been obtained by growing Gd on a Mo(112) substrate. The half filled $4 f$ shell of gadolinium results in highly localized moments which couple via " $R K K Y$-like" interactions through itinerant valence electron states. The magnetic structure of the system is strongly influenced by the intraatomic $4 f-(5 d, 6 s)$ wave function overlap and $5 d, 6 s$ valence electron itinerancy. ${ }^{10}$ The electronic valence-band structure, hybridization, and electron itinerancy are all very sensitive to the lattice configuration. Expansive strain of $4 \%$ within the hexagonal closed-packed system substantially alters the electronic and magnetic valence-band structure. ${ }^{2,3,6}$ The strain results in increased electron localization of the itinerant valence electrons and therefore enhances local moment ferromagnetism.
The thickness dependent Curie temperature of strained $\mathrm{Gd}$, as determined from spin-polarized photoemission, exhibits a decrease with decreasing film thickness, characteristic of finite size scaling behavior. Figure 1 shows the reduced Curie temperature $\left[T_{C}(\infty)-T_{C}(d)\right] / T_{C}(\infty)$ as a function of film thickness $d$ in a logarithmic plot. The data points displayed are for the strained $\mathrm{Gd}$ surface $(\bigcirc)\left[T_{C}(\infty)\right.$ $=370 \mathrm{~K}]$ and the strained Gd bulk $(\boldsymbol{\square})\left[T_{C}(\infty)=340 \mathrm{~K}\right]$. Each data point has been determined from the temperaturedependent exchange splitting and spin polarization of the corresponding spin-polarized photoemission features. While there is a wave vector dependence of the exchange splitting and spin polarization, ${ }^{2}$ the Curie temperatures have been deduced from the valence-band spectra with maximum exchange splitting, which is at the Brillouin zone edge. ${ }^{2}$ This Brillouin zone point provides the best indicator of $T_{C}$. For comparison, the data for unstrained $\operatorname{Gd}(0001)(\bullet)\left[T_{C}(\infty)\right.$ $=293 \mathrm{~K}]$ is shown, which has been extracted from magnetic susceptibility measurements by Farle and co-workers for $\mathrm{Gd} / \mathrm{W}(110) .{ }^{11}$ In this logarithmic plot the slope of the displayed data points marks the critical exponent $\lambda$ of the finite size scaling description of the correlation length, as denoted in Eq. (1).

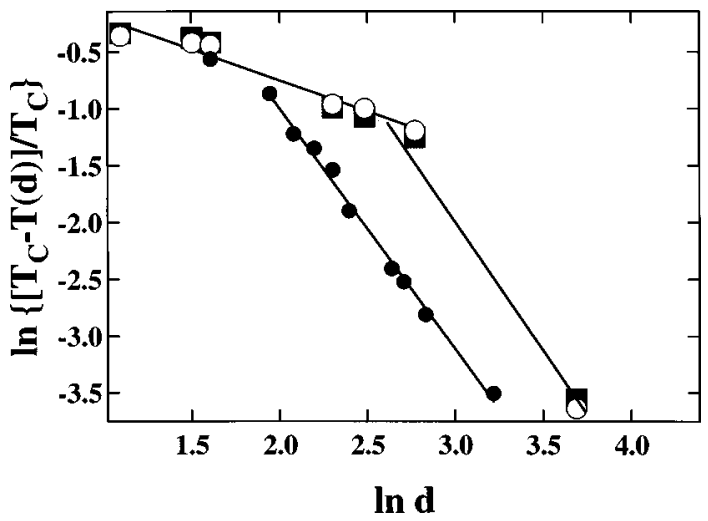

FIG. 1. The reduced Curie temperature $\left[T_{C}(\infty)-T_{C}(d)\right] / T_{C}(\infty)$ as a function of film thickness $d$ in a logarithmic plot. The data points displayed are for the strained Gd surface $(\bigcirc)\left[T_{C}(\infty)=370 \mathrm{~K}\right]$, the strained Gd bulk $(\boldsymbol{\square})$ $\left[T_{C}(\infty)=340 \mathrm{~K}\right]$, and the unstrained $\mathrm{Gd}(\bigcirc)\left[T_{C}(\infty)=293 \mathrm{~K}\right]$. The latter data are adapted from Ref. 11 . 


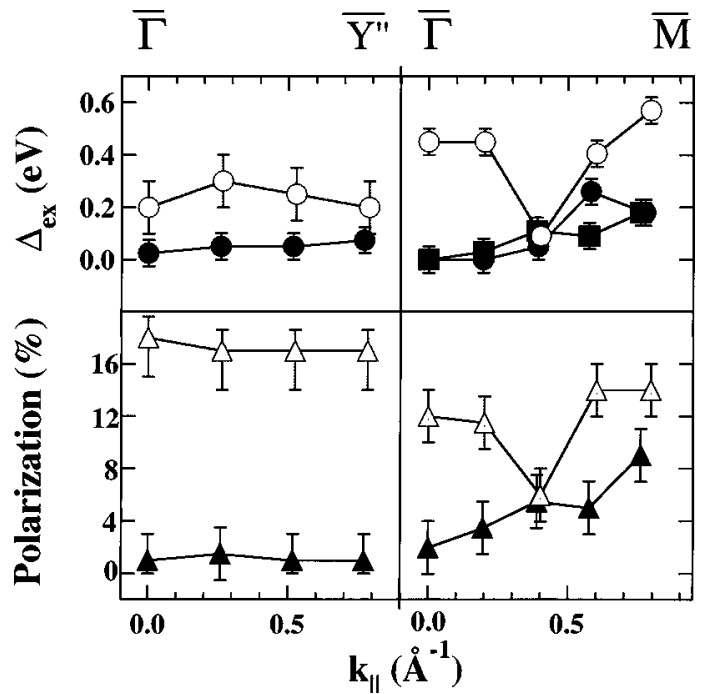

FIG. 2. The exchange splitting (top) and spin asymmetry above background (bottom) of the surface (open symbols) and bulk (filled symbols) spin subbands as a function of wave vector. Data are shown for a 4 ML thick (left) and a 40 ML thick (right) strained Gd film. The data points have been extracted from spin-polarized photoemission spectra.

Clearly the thickness dependence of the reduced Curie temperature for strained thin films of Gd does not follow the expected fixed exponential. A variation of the critical exponent with film thickness is indicated. For film thicknesses of more than approximately 15 monolayers (ML), the critical exponent $\lambda$ is comparable to the one of the unstrained Gd films, as determined by the similar slope. For the ultrathin films $(d \leqslant 15 \mathrm{ML})$, the slope for strained Gd is reduced by a factor of approximately 4 , reflecting a reduced critical exponent $\lambda$ and decreased sensitivity to finite size scaling. A variation of the critical exponent similar to the one evident for strained $\mathrm{Gd}$ is possibly indicated for the unstrained $\mathrm{Gd}$ films at thicknesses of less than approximately $8 \mathrm{ML}$ (Fig. 1). The limited data points for the ultrathin films ( $d$ $<10 \mathrm{ML}$ ) that are presented in Ref. 11 make this postulate, however, rather weak. A deviation from the expected finite size scaling behavior is also apparent in $\mathrm{Gd} / \mathrm{W}$ multilayers for Gd interlayer thickness of less than approximately 15 ML. ${ }^{12}$ A crossover from three- to two-dimensionality is possible, ${ }^{5,13}$ but generally dimensionality crossover should result in an increase in the critical exponent ${ }^{14}$-not a decrease as observed here.

Concomitant with the variation of the critical exponent for decreasing film thicknesses, the bulk bands of the strained Gd films become paramagnetic-like over an increasingly larger volume in $k$-space. ${ }^{3}$ The dominance of the surface magnetic ordering in the ultrathin films is indicated in Fig. 2. Figure 2 plots the exchange splitting (top) and spin asymmetry (bottom) of surface (open symbols) and bulk (filled symbols) spin subbands as a function of wave vector for a 4 ML thick film (left) and a 40 ML thick film (right). While the magnitude of the measured exchange splitting is an indirect indication of the $5 d$ moment, ${ }^{15}$ the polarization (above background) provides insight into the extent of the magnetic activity, Stoner-like magnetism and/or rigid band ferromagnetism.
The exchange splitting of the bulk bands of the $4 \mathrm{ML}$ thick strained Gd films is small throughout the surface Brillouin zone and exceeds the experimental resolution of 0.05 $\mathrm{eV}$ only near the zone edge $\left(\Delta_{\mathrm{ex}} \approx 0.07 \mathrm{eV}\right)$, indicative of minimal Stoner-like ferromagnetism, away from the vicinity of the zone edge $\bar{Y}^{\prime \prime}$. There is also no significant polarization (above background) in the region of the Gd bulk bands well away from the Fermi level for any wave vector. The bulk bands of the 4 ML thick Gd films exhibit the paramagneticlike behavior expected with direct contact to a ferromagnet. For the $40 \mathrm{ML}$ thick films, the magnetic ordering of the bulk bands follows a distinct wave vector dependence with negligible exchange splitting at the zone center $\bar{\Gamma}$ and substantial exchange splitting of approximately $0.25 \mathrm{eV}$ near the zone edge $\vec{M}$. The spin asymmetry behaves similarly with minimal asymmetry at $\bar{\Gamma}$ and large polarization at $\bar{M}$. The wave vector dependent exchange splitting of the thicker strained Gd films suggests a change from paramagnetic-like behavior at the Brillouin zone center to Stoner-like magnetism at the zone edge.

The magnetic ordering of the surface behaves differently from that of the bulk. The exchange splitting of the surface state of the $4 \mathrm{ML}$ thick Gd films is of the order of $0.25 \mathrm{eV}$ with little variation for different wave vectors. The spin polarization in the region of the surface sensitive states approaches approximately 17\% (above background). The large spin asymmetry and relatively small exchange splitting is indicative of predominantly rigid band magnetism with some Stoner-like ferromagnetic contributions, largely independent of wave vector. This is in contrast to the strong wave vector dependence of the magnetic behavior of the thicker films ( 40 ML). The surface state exchange splitting is large at the Brillouin zone center $\left(\Delta_{\mathrm{ex}} \approx 0.45 \mathrm{eV}\right)$ and at the Brillouin zone edge $\left(\Delta_{\mathrm{ex}} \approx 0.57 \mathrm{eV}\right)$. The minimum in the exchange splitting of the surface feature at the Brillouin zone interior $\left(\Delta_{\mathrm{ex}} \approx 0.10 \mathrm{eV}\right)$ has been attributed to a wave vector dependent change from a surface state (zone center and zone edge) to a surface resonance (zone interior). ${ }^{2}$ This dip of the surface exchange splitting is reflected in the spin asymmetry, which reduces to $4 \%$ in the Brillouin zone interior as compared to $10 \%$ at the zone center and $12 \%$ at the zone edge.

With decreasing film thickness the region of the Brillouin zone in which the bulk bands are paramagnetic becomes increasingly larger and the surface magnetic ordering increasingly dominant, characterized by diminishing Stonerlike magnetism and strengthened rigid band magnetism. At the same time the bandstructure of the strained Gd films changes significantly. The bulk bands of the thicker films are dispersive with a bandwidth $w$ of approximately $1.6 \mathrm{eV}$, in contrast to the ultrathin films which appear nearly dispersionless $(w \leqslant 0.15 \mathrm{eV})$. This has substantial consequences for the coherence length which can be estimated as $l$ $\approx \sqrt{w / k_{B} T_{C}} a .{ }^{16}$ Considering that there is a change in the bandwidth $w$ of a factor of approximately 10 with decreasing film thickness, the bulk electron coherence length in the ultrathin films is expected to be much shorter as compared to the thicker films. The surface state electrons of the strained Gd films are considerably more localized as compared to 
those of the bulk bands, which is reflected in the narrow bandwidth of $w \leqslant 0.2 \mathrm{eV}$, which is even smaller for the thinner film thicknesses. As a result, the magnetic structure of the increasingly thinner strained Gd films are governed by an $R K K Y$ interaction that is of increasingly shorter electron coherence length. Finite size effects may become less influential for decreased electron coherence lengths well away from $T_{C}$. In any case, the surface state exchange splitting is large at the Brillouin zone center where the band structure is most sensitive to long range order. Some bulk bands exhibit the smallest exchange splitting at zone center. This indicates that the surface may increasingly dominate the thin film magnetism. A deviation from finite size scaling in terms of a reduced critical exponent is expected and consistent with our thickness dependent critical temperature data (Fig. 1). For the limit of $5 d$ valence electrons that are highly localized, polarized only by the local $4 f$ moments (which themselves do not have to be aligned, due to spin wave excitations), the electron coherence length becomes negligible and a vanishing critical exponent $(\lambda \rightarrow 0)$ is expected for the thin film limit $(d \rightarrow 1)$, a change distinct from dimensionality effects.

The reduced critical exponent for the ultrathin films is consistent with the magnetic behavior observed in free $\mathrm{Gd}$ clusters by Douglass et al. ${ }^{17,18} \mathrm{Gd}$ clusters of different sizes exhibit very similar magnetic moments per atom (although substantially reduced from that of the bulk gadolinium value). More importantly the Curie temperatures of these small $\mathrm{Gd}_{N}$ clusters $(N=10-92)$ are even larger than that of bulk gadolinium. Clearly, finite size scaling behavior fails in the physical world of small clusters and a trend of $\lambda \rightarrow 0$ as $N \rightarrow 1$ is indicated. Indeed ferromagnetic monolayers at finite temperature are known and routinely observed in the thin film limit of ultrathin films. Cluster studies indicate that the atomic coordination number may have significant influence on the magnetic behavior, and are an example of a catastrophic failure of finite size scaling behavior.

In the thin film limit, the apportionment of relative roles in the magnetic ordering of surface and bulk becomes very important for the description of the finite size scaling behav- ior. For strained Gd films, the short electron coherence length of the $R K K Y$ interaction is dominating the magnetic behavior of the ultrathin films, with the result of a substantially reduced critical exponent $\lambda$. There is no a priori reason why this behavior should be restricted to the strained $\mathrm{Gd}$ films grown on a $\mathrm{Mo}(112)$ substrate. In fact a similar behavior is anticipated in the "relatively unstrained" $\operatorname{Gd}(0001)$ ultra-thin films grown on W(110), although experimental confirmation does not as yet exist.

This work was supported by NSF through Grant Nos. DMR-92-21655 and DMR-94-96131. The experiments were carried out at the National Synchrotron Light Source which is funded by the DOE.

${ }^{1}$ M. Donath, in Electronic Surface and Interface States on Metallic Systems, edited by E. Bertel and M. Donath (World Scientific, Singapore, 1995), p. $233 \mathrm{ff}$

${ }^{2}$ C. Waldfried, T. McAvoy, D. Welipitiya, P. A. Dowben, and E. Vescovo, Phys. Rev. B (submitted).

${ }^{3}$ C. Waldfried, T. McAvoy, D. Welipitiya, E. Vescovo, and P. A. Dowben, Europhys. Lett. (submitted).

${ }^{4}$ M. E. Fisher and M. N. Barber, Phys. Rev. Lett. 28, 1516 (1972).

${ }^{5}$ A. Aspelmeier, F. Gerhardter, and K. Baberschke, J. Magn. Magn. Mater. 132, 22 (1994); J. T. Ou, F. Wang, and D. L. Lin, Phys. Rev. E 56, 2805 (1997).

${ }^{6}$ C. Waldfried, D. N. Mcllroy, and P. A. Dowben, J. Phys.: Condens. Matter 9, 10615 (1997)

${ }^{7}$ C. Waldfried, D. N. Mcllroy, and P. A. Dowben, Phys. Rev. B 54, 16460 (1996); 56, 9973 (1997)

${ }^{8}$ C. Waldfried, D. N. Mcllroy, D. Welipitiya, T. McAvoy, E. Vescovo, and P. A. Dowben, J. Appl. Phys. 83 (1998).

${ }^{9}$ D. Li, J. Pearson, S. D. Bader, D. N. Mcllroy, C. Waldfried, and P. A. Dowben, Phys. Rev. B 51, 13895 (1995).

${ }^{10}$ W. Nolting, T Dambeck, and G. Borstel, Z. Phys. B 94, 409 (1994).

${ }^{11}$ M. Farle et al., Phys. Rev. B 47, 11571 (1993).

${ }^{12}$ J. S. Jiang and C. L. Chien, J. Appl. Phys. 79, 5615 (1996).

${ }^{13}$ Yi Li and K. Baberschke, Phys. Rev. Lett. 68, 1208 (1992).

${ }^{14}$ K. Binder and P. C. Hohenberg, Phys. Rev. B 9, 2194 (1973); T. Kaneyoshi, Introduction to Surface Magnetism (CRC, Boca Raton, FL, 1991).

${ }^{15}$ F. J. Himpsel, J. Magn. Magn. Mater. 102, 261 (1991).

${ }^{16}$ H. Capellmann and V. Vieira, Solid State Commun. 43, 747 (1982).

${ }^{17}$ D. C. Douglass, J. P. Bucher, and L. A. Bloomfield, Phys. Rev. Lett. 68 , 1774 (1992).

${ }^{18}$ D. C. Douglass, A. J. Cox, J. P. Bucher, and L. A. Bloomfield, Phys. Rev. B 47, 12874 (1992). 